\title{
Directed Hypergraph-based Models for the Fault Monitoring of Chemical Reaction Kinetics
}

\author{
H. Ajemni \\ MACS Laboratory: Modeling, Analysis and Control of Systems \\ National Engineering School of Gabes (ENIG) \\ University of Gabes, Tunisia
}

\author{
R. El Harabi \\ MACS Laboratory: Modeling \\ Analysis and Control of Systems \\ National Engineering School of Gabes (ENIG) \\ University of Gabes, Tunisia
}

\author{
M. N. Abdelkrim \\ MACS Laboratory: Modeling \\ Analysis and Control of Systems \\ National Engineering School of Gabes (ENIG), \\ University of Gabes, Tunisia
}

\begin{abstract}
The paper deals with the use of directed hypergraph so as to model chemical reaction kinetics. Firstly, the model is directly deduced both from using systematic generation from chemical equation laws, then, based on the Bond Graph-Hypergraph analog given in details. Secondly, the causal and structural properties of a directed hypergraph are involved to design the Fault Detection and Isolation (FDI) algorithm scheme by generating redundancy relations through covering causal hyperpaths. The integrated framework is demonstrated to be effective through a tutorial example of via a reaction with a second order.
\end{abstract}

\section{General Terms}

Graphical Modelling, Fault Diagnosis, Chemical reactions

\section{Keywords}

Directed hypergraph, chemical reaction kinetics, structural analysis, fault monitoring

\section{INTRODUCTION}

Modeling of physical systems with all engineering fields is a helpful tool to understand the behavior of the system without having to experiment on it which is very important at industrial level. When compared with other engineering branches, a chemical process engineering is characterized by the complexity and the difficulty in obtaining convenient dynamical models. This result from the fact that they are based on chemical and physical principals that include different branches of science such as thermodynamics, physical chemistry, fluid mechanics, heat transfer, mass transfer, reaction kinetic, etc.

Most of the chemical processes can be modelled as a system of algebraic equation (AE), differential equation (DE) or differential algebraic equation (DAE). Mathematical model of chemical pro- cesses are helpful tools for the development of processes and control schemas. However, due to the strong non linearities and parameters uncertainties in the chemical systems the formulation of these models themselves is still an obstacle for the application of modelbased techniques in industry.

In this context, the behavior of reaction kinetics is determined by structural constraints on mass and energy balances and follows from the stoichiometry of the reaction. Thus, the graphical model such as Bond Graph (graphical quantitative model) and Signed Directed Graph (graphical qualitative model) tools become significant in this case because they are appropriate for the multi-physics modeling of complex systems. Therefore, various techniques [10] [5] have been previously used to model the thermodynamic phenomena in the reaction. Research works [7] show how to use (Pseudo) Bond Graph to model chemical systems.

Like in many areas of sciences [9] [2], hypergarphs can be useful in chemical engineering. Directed Hypergraphs have been recently used for modeling chemical reaction networks [12],[11].. As stated by Zeigarnik [12], the multimolecular reactions are modelled by using directed hypergraphs. For example protein complexes, providing associations between one, two or more proteins illustrate the difference that may arise in modeling biological facts with graphs and hypergraphs. For chemical reaction networks, co-authors [11] describe a reaction by a weighted directed hyperedge where nodes are the chemical and hyperedges are the reactions. All of these applications and other [8] indicate that the Hypergraph offers a framework which helps to overcome such conceptual limitations.

From above-mentioned, how to deal with the modelling chemical reaction kinetics based on hypergraph tools is not yet developed in the literature. Moreover, the current results are not sufficient, which inspires the present investigation.

Motivated by the aforementioned analysis, the representation of chemical reaction kinetics using hyper-graphs comes from the the fact that the model is obtained based on the material and energy balances which do not include only binary relations. The present paper aims to increase communities' awrarness of hypergraphs as a mod- 
eling framework for chemical reaction kinetic phenomenon. This graphical model is based on energy, material and species balances. To proof the obtained model, Bond graph-Hypergraph analogy is detailed.

The remainder of this paper is organized as follows: Section 2 presents the problem formulation, notations and preliminaries related to Bong Graph and Hypergraph formalisms and we conclude this section by an illustrative example of modeling with graphs (BG and directed hypergraph). Section 3 provides the two proposed modeling approaches using hypergraph tool. The new hypergraph model based for FDI design is developed in section 4. The developed methodology is applied in section 5 for a reaction with a second order and we give a comparative study highlighting the strength of the use of hypergraph modeling in section 6. Finally, section 7 concludes the works and gives some outlooks.

\section{PROBLEM FORMULATION, NOTATIONS AND PRELIMINARIES}

\subsection{Problem formulation}

We consider a reaction with a set $E$ of educts , a set $P$ of products and stoichiometric coefficients $v_{i}$ :

$$
v_{A_{1}} A_{1}+\cdots+v_{A_{n}} A_{n} \underset{A_{r}}{\stackrel{A_{f}}{\rightleftharpoons}} v_{B_{1}} B_{1}+\cdots+v_{B_{m}} B_{m}
$$

where $\bigcup_{i=1}^{n} A_{i}=E, \bigcup_{j=1}^{m} B_{j}=P$,

The reaction rate follows a mass-action low:

$$
J=k_{+} \prod_{l \in E} C_{l}^{\left|v_{l}\right|}-k_{-} \prod_{l \in P} C_{l}^{\left|v_{l}\right|}
$$

with constant coefficients $k_{+}$and $k_{+}$.

As can be derived from the fundamental equations of thermodynamics and the mole balances, the density of entropy production $\dot{S}_{g e n}\left[J K^{-1} m^{-3} s^{-1}\right]$ of a reaction is given by:

$$
A * J=T * \dot{S}_{g e n}
$$

where $J\left[\mathrm{molm}^{-3} \mathrm{~s}^{-1}\right]$ is the reaction flux, $T[K]$ the thermodynamic temperature and $A\left[\mathrm{Jmol}^{-1}\right]$ is the negative Gibbs reaction energy:

$$
A=A_{f}-A_{r}
$$

where $A_{f}$ and $A_{r}$ are linear combinations of the chemical potentials $\mu_{i}$ :

$$
\begin{aligned}
& A_{f}=\sum_{i \in E}\left|v_{i}\right| \mu_{i} \\
& A_{r}=\sum_{i \in P}\left|v_{i}\right| \mu_{i}
\end{aligned}
$$

For ideal mixtures, the chemical potentials $\mu_{i}$ are given by :

$$
\mu_{i}=\mu_{0}^{i}+R T \ln \left(C_{i} / C_{0}\right)
$$

with $C_{l}=\frac{n_{l}}{V}=\frac{1}{V} \int \dot{n}_{l}$, where $V$ the volume of the reaction, $\mu_{0}^{i}$ the chemical standard potential of pure chemical species , $C_{0}$ a standard concentration (e.g. $C_{0}=1 \mathrm{~mol} / L$ ) and $R$ the universal gas constant. Thus, we obtain:

$$
\mu_{i}=\mu_{0}^{i}+R T \ln \left(\frac{1}{C_{0} V} \int \dot{n}_{l}\right)
$$

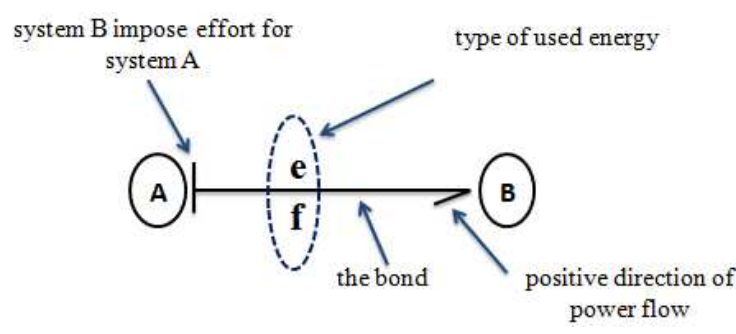

Fig. 1. BG definition and causality concept

As we can write :

$$
J=k_{+} \prod_{i \in E}\left(\frac{\int \dot{n}_{l}}{V}\right)^{\left|v_{i}\right|}-k_{-} \prod_{i \in P}\left(\frac{\int \dot{n}_{l}}{V}\right)^{\left|v_{i}\right|}
$$

The molar balances of different species are defined as the following

$$
\left\{\begin{array}{l}
\dot{n}_{l}=\dot{n}_{l i}-\dot{n}_{l o}-\dot{n}_{1 l} \quad, \quad l=A_{i}, i=1 \cdots n \\
\dot{n}_{l}=-\dot{n}_{l o}+\dot{n}_{1 l} \quad, \quad l=B_{j}, j=1 \cdots m
\end{array}\right.
$$

such that $\dot{n}_{1 l}=v_{l} J$ and $\dot{n}_{l i}$ and $\dot{n}_{l o}$, respectively denote the inlet and outlet molar flow, are written by:

$$
\left\{\begin{array}{l}
\dot{n}_{l i}=\dot{m}_{l} / M_{l} \quad, \quad l=A_{i} \\
\dot{n}_{l o}=n_{l}\left(\dot{m}_{l} /(\rho V)\right) \quad, \quad l=A_{i}, B_{j}
\end{array}\right.
$$

$\rho$ : the mass density, $M_{l}$ : the molar mass.

The understanding of chemical kinetics rate is a fundamental issue in chemical engineering. A detailed mathematical model is difficult or is unavailable. Therefore, graphs are suitable tools for modeling chemical processes. But, a key properties of graphs is that every edge connects exactly two nodes and many chemical relations are characterized by more than two participating partners and are thus multilateral. Hyper-graph offers a framework to overcome such limitations. In mathematics, a hypergraph is a generalization of graph where edges can connect any number of vertices. Using the characters of hyper-graph that edge is represented as multi-set of nodes, we propose hyper-graph as a natural way to represent systems.

\subsection{Notations and preliminaries}

2.2.1 Basic element of Bond graph. Since details of Bond Graph theory can be easily found in [10] this section briefly recalls coupled Bond Graph representation for modeling of chemical process. Bon graph can be considered as a graph $G=(V, E)\}$ whose nodes $V$ represents subsystems basic elements (junction) or components and edges $E$ show the power transfers within a system (power bonds). As shown in Figure 1 Bond Graph models are composed of multiports related by power bonds representing the identity between a pair of conjugated variables (named effort $(e)$ and flow $(f)$ ) whose product is the instantaneous energy flow between the multiport elements. Effort is the intensive variable (e.g. pressure,electrical potential, chemical potentiel, temperature...) and flow is the derivative of the extensive variable (e.g. volume flow, current,velocity, entropy flow...). Effort variable is labeled above the bond and flow variable is labeled below the band.The positive direction of power flow (a generalized coordinate system used in bond graph models) is represented by the half-arrow on the bond. This power is the product of the two power variables.

The determination of causes and effects (by a covering causal path) 


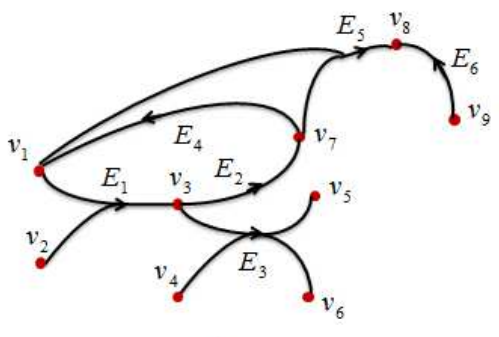

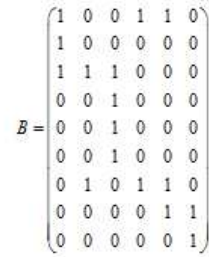

Fig. 2. directed hypergraph and its incidence matrix

in the system is directly deduced from the graphical representation. The causality is denoted by a cross-stroke (see Figure 11). By convention, the causal stroke is placed near (respectivelyfar from) the bond graph element for which the effort (respectively flow) in known.

2.2.2 Fundamentals of Hypergraph. For basic definitions of hypergraph we refer the reader to [3].A hypergraph is a generalization of a graph where an edge can be connected to any number of vertices. formally a hypergaph $H(V, E)$ consists of two sets $V=\left(v_{1}, v_{2}, \ldots, v_{n}\right)$ is the set of vertices and $E=\left(e_{1}, v_{2}, \ldots, e_{m}\right)$ is the set of hyperedges where $e_{i} \subseteq V$ for $i=1 \ldots m$.

$A=\left[a_{i j}\right]$ a $n \times m$ matrix such that $a_{i j} \in\{0,1\}$ defines the incidence matrix of the hypergraph $H$ where each row $i$ is associated with a vertex $v_{i}$ and each column $j$ is associated with an hyperedge $e_{j}$.

2.2.3 Basic directed Hypergraph Concepts. A directed hypergraph is a pair $(\mathrm{V}, \mathrm{E})$ where $V$ is a finite set of vertices and $E \subseteq 2^{V} * 2^{V}$ is a set of hyperarcs (hyperedge), such that every $e=(T(e), H(e)) \in E$ is an ordered pair of non-empty disjoint subsets $T(e)$ and $H(e)$. where $T(e)$ and $H(e)$ are the sets of vertices that appear respectively in the tail and the head of the hyperarc e. We say that e is incident on each vertex in $T(e) \cup H(e)$. For each $v \in T(e), e$ is outgoing hyperedge from $v$ and for each $v \in H(e), e$ is incoming hyperedge to $v$.

An $E_{1} \subset E$ induced a directed hypergraph $H_{1}\left(V_{1}, E_{1}\right)$ of $H(V, E)$ is defined as directed hypergraph with $V_{1}=\left(\cup_{e \in E_{1}}\right.$ head $\left.(e)\right) \cup\left(\cup_{e \in E_{1}}\right.$ tail $\left.(e)\right)$

$H_{1}=\left(V_{1}, E_{1}\right)$ is called a subhypergraph of the hypergraph $H$ such that $E_{1} \subset E$ and $V_{1} \subset V$.

As for directed graphs, directed hypergraph is specified with its adjacency matrix $A$ and its incidence matrix $B$ :

Two vertices of hypergraph are said to be adjacent, if there is an edge that contains both of these vertices. Two edges are said to be adjacent, if their intersection is not empty(i.e. there is a vertex adjacent to both edges).

Adjacency matrix A of a directed hypergraph is a square symmetric matrix whose entries $a_{i j}$ are the number of hyper-edges that contain both nodes $v_{i}$ and $v_{j}$, the diagonal entries of A are zero. In the incidence matrix $B$ each of the rows is associated with a vertex and each of the columns is associated with an hyper-edge, where $b_{i j}=1$ if $v_{i} \in H\left(e_{j}\right), b_{i j}=-1$ if $v_{i} \in T\left(e_{j}\right)$ and $b_{i j}=0$ otherwise.

The adjacency matrix is related to the incidence matrix by the following theorem $A=B B^{T}-D$ where $B^{T}$ is the transpose of the incidence matrix and $D$ is the diagonal matrix whose diagonal entries are the degrees of the vertices.

Figure 2 (part a) shows a directed hypergraph $H$ with nine vertices $\left\{v_{1}, v_{2}, v_{3}, \ldots, v_{9}\right\}$ and six hyperedges $\left\{E_{1}, E_{2}, E_{3}, E_{4}, E_{5}, E_{6}\right\}$,

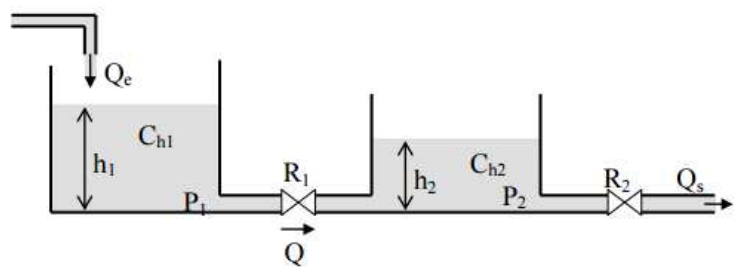

Fig. 3. hydraulic two tank system

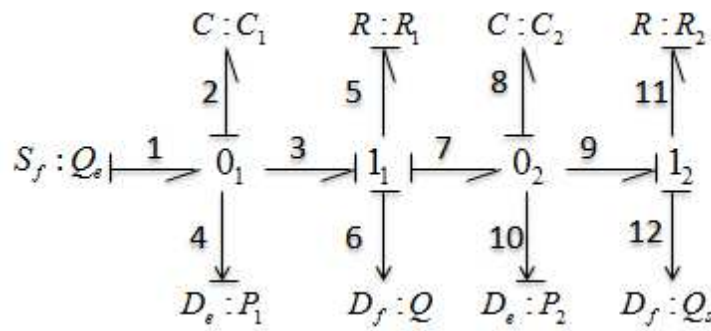

Fig. 4. BG model of the hydraulic two tank system

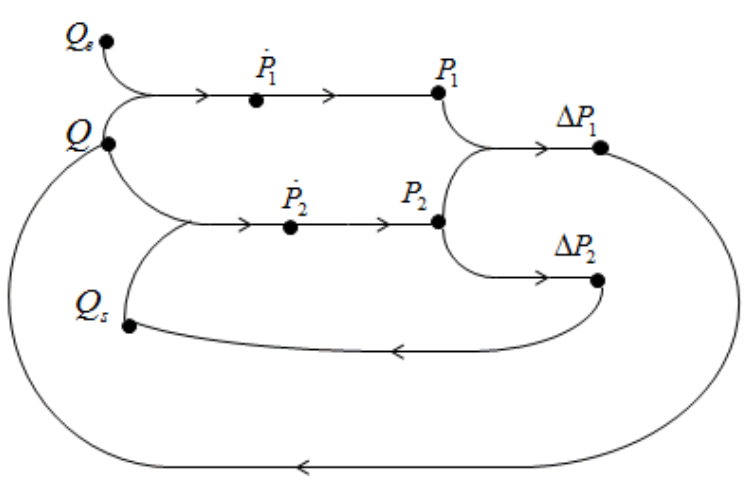

Fig. 5. Hypergraph model of the hydraulic two tank system

where:

$E_{1}=\left(\left\{v_{1}, v_{2}\right\},\left\{v_{3}\right\}\right), E_{2}=\left(\left\{v_{3}\right\},\left\{v_{7}\right\}\right)$,

$E_{3}=\left(\left\{v_{3}, v_{4}\right\},\left\{v_{5}, v_{6}\right\}\right), E_{4}=\left(\left\{v_{7}\right\},\left\{v_{1}\right\}\right), E_{5}=\left(\left\{v_{1}, v_{7}\right\},\left\{v_{8}\right\}\right)$ and $E_{6}=\left(\left\{v_{9}\right\},\left\{v_{8}\right\}\right)$

The corresponding incidence matrix is shown in (part b).

A simple hyperpath $P_{s t}$ of length $q$, in directed hypergrath $H=(V, E)$, is a sequence $\left(v_{1}, e_{1}, v_{2}, e_{2}, \ldots, v_{q}, e_{q}, v_{q+1}\right)$ consisting of distinct vertices $v_{i}$, where $1 \leq i \leq q+1$, and distinct hyperedges $e_{j}, 1 \leq j \leq q$, such that $s=v_{1}, t=v_{q+1}$, and for every $1 \leq j \leq q, v_{i} \in T\left(e_{i}\right)$ and $v_{i+1} \in H\left(e_{i}\right)$.

The forward star (FS) and the backward star (BS) of a vertex $v_{i}$ are ,respectively, defined by $F S\left(v_{i}\right)=\{e \in E, v \in T(E)\}$ and $B S\left(v_{i}\right)=\{e \in E, v \in H(E)\}$.

2.2.4 Modeling with Graphs: Illustrative example . Let us consider a simple hydraulic system consisted of two tanks depicted in Figure 3 where $Q_{e}$ is the input flow, $Q$ and $Q_{s}$ are, respectively, the outputs flow in tank 1 and tank 2 and $h_{1}$ and $h_{2}$ are, respectively, the level-tank 1 and the level-tank2.This system is defined by the 
following differential equations :

$$
\left\{\begin{array}{c}
C_{h 1} \frac{d P_{1}}{d t}=Q_{e}-Q \\
C_{h 2} \frac{d P_{2}}{d t}=Q-Q_{s} \\
Q=\frac{P_{1}-P_{2}}{R_{1}}=\frac{\Delta P_{1}}{R_{1}} \\
Q_{s}=\frac{P_{2}}{R_{2}}=\frac{\Delta P_{2}}{R_{2}}
\end{array}\right.
$$

such that $P_{1}=\gamma h_{1}$ and $P_{2}=\gamma h_{2}, C_{h 1}, C_{h 2}$ are respectively the hydraulic capacitance of tank 1 and tank 2 and $R_{1}$ and $R_{2}$ are, respectively, the hydraulic resistance of tank 1 and tank 2 .

Based on Equation (12), the BG and hypergraph models of the hydraulic system are given respectively by Figure (4) and Figure 5. The hypergraph model $H=(V, E)$ is composed by nine vertices $V=\left\{Q_{e}, Q, Q_{s}, P_{1}, P_{2}, \dot{P}_{1}, \dot{P}_{2}, \Delta P_{1}, \Delta P_{2}\right\}$ and eight hyperedges $E=\left\{E_{1}, E_{2}, \ldots, E_{8}\right\}$ where $E_{1}=\left\{\left\{Q_{e}, Q\right\},\left\{\dot{P}_{1}\right\}\right\}, E_{2}=$ $\left\{\left\{\dot{P}_{1}\right\},\left\{P_{1}\right\}\right\}, E_{3}=\left\{\left\{Q_{s}, Q\right\},\left\{\dot{P}_{2}\right\}\right\}, E_{4}=\left\{\left\{\dot{P}_{2}\right\},\left\{P_{2}\right\}\right\}, E_{5}=$ $\left\{\left\{P_{1}, P_{2}\right\},\left\{\Delta P_{1}\right\}\right\}, E_{6}=\left\{\left\{P_{2}\right\},\left\{\Delta P_{2}\right\}\right\}, E_{7}=\left\{\left\{\Delta P_{1}\right\},\{Q\}\right\}$ and $E_{8}=\left\{\left\{\Delta P_{2}\right\},\left\{Q_{s}\right\}\right\}$

\section{PROPOSED HYPER-GRAPH REPRESENTATION FOR CHEMICAL REACTIONS}

\subsection{Systematic procedure to derive a hyper-graph model}

In this section, we propose a hypergraph model for the chemical reaction rate (Global hypergraph) which is a collection of six subhypergraph of different phenomena of chemical reactions. Details related to each of these subhypergraphs are listed as follows:

3.1.1 The molar balance. Equation (10) may be modeled as a subhypergraph $H_{n}=\left(V_{n}, E_{n}\right)$ with molar flow $\dot{n}$, inlet and outlet molar flow $\dot{n}_{l i}, \dot{n}_{l o}$ of different species corresponding to vertices and relations which assembled them corresponding to hyperedges. It is defined as follows :

$$
V_{n}=\left\{\dot{n}_{l}, \dot{n}_{1 l}, \dot{n}_{k i}, \dot{n}_{l o}, k=A_{i}, l=A_{i}, B_{j}, i=1 \cdots n, j=1 \cdots m\right\}
$$

$V_{n}$ a finite set of vertices and

$$
E_{n}=\left\{E_{l}^{1 n}, E_{k}^{2 n}\right\}
$$

$E_{n}$ a set of non empty subsets of $V_{n}$ such that:

$$
\begin{aligned}
& E_{l}^{1 n}=\left\{\left(\left\{\dot{n}_{l}\right\},\left\{\dot{n}_{l i}, \dot{n}_{l o}, \dot{n}_{1 l}\right\}\right), l=A_{i}, i=1 \cdots n\right\} \\
& E_{k}^{2 n}=\left\{\left(\left\{\dot{n}_{k}\right\},\left\{\dot{n}_{k o}, \dot{n}_{1 k}\right\}\right), k=B_{j}, j=1 \cdots m\right\}
\end{aligned}
$$

Note that $E_{n} \subseteq 2^{V_{n}}, E_{l}^{1 n} \cup E_{k}^{2 n}=V_{n}$

3.1.2 The reaction flux. We may represent equation 9 ) by a subhypergraph $H_{r f}=\left(V_{r f}, E_{r f}\right)$ with $n+m$ molar flow vertices and a B-hyperedge $(|H(e)=1|)$ consisting of that equation. $H_{r f}$ is defined through the following sets:

$$
\begin{gathered}
V_{r f}=\left\{J, \dot{n}_{l}, l=A_{i}, B_{j}, i=1 \cdots n, j=1 \cdots m\right\} \\
E_{r f}=\left\{\left\{\dot{n}_{l}\right\},\{J\}\right\}
\end{gathered}
$$

3.1.3 The chemical potential. Our model views the chemical potential (equation(9)) as a subhypergraph $H_{\mu}=\left(V_{\mu}, E_{\mu}\right)$ where we represent each molar flow $\dot{n}_{l}$ and chemical potential $\mu_{l}$ of all species by a vertex and relation assembling them by a hyperedge. The sets $V_{\mu}$ and $E_{\mu}$ of vertices and hyperedges respectively are given as:

$$
\begin{aligned}
& V_{\mu}=\left\{\dot{n}_{l}, \mu_{l} \quad, l=A_{i}, B_{j}, i=1 \cdots n, j=1 \cdots m\right\} \\
& E_{\mu}=\left\{E_{\mu l}, l=A_{i}, B_{j}, i=1 \cdots n, j=1 \cdots m\right\} \subseteq 2^{V_{\mu}} \\
& \text { where } E_{\mu l}=\left\{\left(\left\{\dot{n}_{l}\right\},\left\{\mu_{l}\right\}\right)\right\} \text { and } \cup E_{\mu l}=V_{\mu}
\end{aligned}
$$

3.1.4 The chemical affinity. In the chemical affinity subhypergraph $H_{A}=\left(V_{A}, E_{A}\right)$, we represent each of chemical potential of different species $\mu_{l}$, forward affinity $A_{f}$, reverse affinity $A_{r}$ and chemical affinity $A$ by a vertex and equations 4,5 and $(6$ by the set of hyperedges:

$$
V_{A}=\left\{\mu_{l}, A_{f}, A_{r}, A \quad, l=A_{i}, B_{j}, i=1 \cdots n, j=1 \cdots m\right\}
$$

$$
E_{A}=\left\{E_{f}, E_{r}, E_{a}\right\}
$$

where:

$$
\begin{gathered}
E_{f}=\left\{\left\{\mu_{A_{i}}\right\},\left\{A_{f}\right\}, i=1 \cdots n\right\} \\
E_{r}=\left\{\left\{\mu_{B_{j}}\right\},\left\{A_{r}\right\}, j=1 \cdots m\right\}, \\
E_{a}=\left\{\left\{A_{f}, A_{r}\right\},\{A\}\right\},
\end{gathered}
$$

with $E_{A} \subseteq 2^{V_{A}}, E_{a} \cup E_{f} \cup E_{r}=V_{A}$

3.1.5 The hydraulic chemical relationship. We use the subhypergraph $H_{c h}=\left(V_{c h}, E_{c h}\right)$ whose vertices are the molar flow $\dot{n}_{l}$, the inlet and outlet molar flow $\dot{n}_{k i}, \dot{n}_{l o}$ and the mass flow $\dot{m}_{l}$, and hyperedges represented by equations 11 . $V_{c h}$ and $E_{c h}$ are defined as follows:

$$
\begin{gathered}
V_{c h}=\left\{\dot{n}_{l}, \dot{n}_{k i}, \dot{n}_{l o}, \dot{m}_{l}, \quad k=A_{i}, i=1 \cdots n, l=A_{i}, B_{j},\right. \\
i=1 \cdots n, j=1 \cdots m\} \\
E_{c h}=\left\{E_{1 \text { ch_k }}, E_{2 c_{\_} l}, k=1 \cdots n, l=1 \cdots n+m\right\}
\end{gathered}
$$

where:

$$
\begin{aligned}
& E_{1 c h \_k}=\left\{\left(\left\{\dot{m}_{l}\right\},\left\{\dot{n}_{l i}\right\}\right), l=A_{i}, i=1 \cdots n\right\} \\
& E_{2 c h \_l}=\left\{\left(\left\{\dot{m}_{l}, \dot{n}_{l}\right\},\left\{\dot{n}_{l o}\right\}\right), l=A_{i}, B_{j}, i=1 \cdots n,\right. \\
& j=1 \cdots m\} \\
& E_{c h} \subseteq 2^{V_{c h}}, E_{1 c h \_l} \cup E_{2 c h \_k}=V_{c h}, \quad k=1 \ldots n, l=1 \ldots n+m
\end{aligned}
$$

3.1.6 The thermal chemical relationship. The thermal chemical relationship is modeled by the subhypergraph $H_{c t}=\left(V_{c t}, E_{c t}\right)$ whose vertices are the chemical affinity $A$, the reaction flux $J$, the temperature $T$ and the entropy flow $\dot{S}$ and a hyperedge represented by equation 3 :

$$
\begin{gathered}
V_{c t}=\left\{A, J, T_{r}, \dot{S}\right\} \\
E_{c t}=\left\{\{A, J\},\left\{T_{r}, \dot{S}\right\}\right\}
\end{gathered}
$$




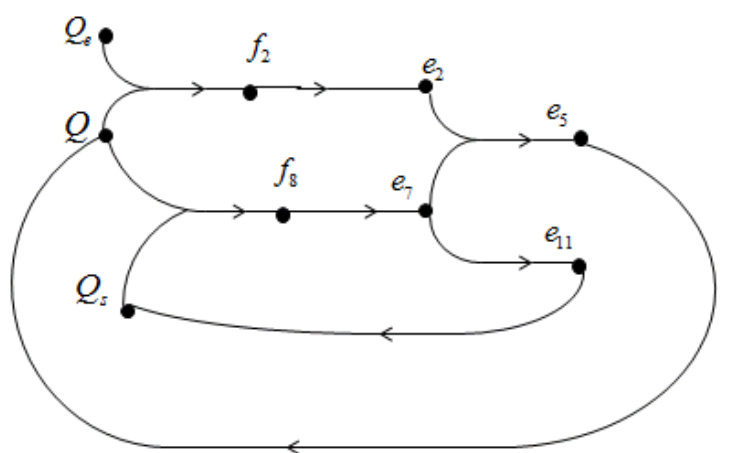

Fig. 6. Hypergraph model obtained by BG-Hypergraph transformations

3.1.7 Global hypergraph. As we defined a chemical reaction by the combination of different phenomena that appear, the global hypergraph $H=(V, E)$ modeling the chemical reaction rate is the association of all the previous subhypergraphs such that:

$$
V=\left\{V_{n} \cup V_{r f} \cup V_{\mu} \cup V_{A} \cup V_{c h} \cup V_{c t}\right\}
$$

the finite set of vertices and

$$
E=\left\{E_{n}, E_{r f}, E_{\mu}, E_{A}, E_{c h}, E_{c t}\right\}
$$

the set of hyperedge of $H$

$E \subseteq 2^{V}, \quad \cup E_{i}=V, \quad i=n, \mu, A, c h, c t$.

\subsection{Bond Graph-Hypergraph transformations}

Built the Bond Graph.To get graphical model systems by developing a hypergraph model directly from the Bond Graph, we propose rules of transformations from the Bond graph to the hypergraph model. In the resulting directed hypergraph, effort and flow variables will be represented as vertices, while relations between variables will be represented as directed hyperedges. The causality results in the arrow of the hyperedge indicating the direction of the power transfer (see [1] for more details).

\subsection{Illustrative example}

In order to show the impact of our BG-Hypergraph transformations, we have used, again, in this section the hydraulic two tank system as an example. The BG model of the studied example is given by Figure 4 . Based on the given rules, the hypergraph model is given by Figure 6 Such that $f_{2}=\dot{P}_{1}, f_{8}=\dot{P}_{2}, e_{2}=P_{1}$ and $e_{5}=P_{1}-P_{2}$, $e_{7}=P_{2}$ and $e_{11}=P_{2}$, one may conclude on the efficiency of the proposed Bond Graphs-Hypergraph analogy.

\section{HYPERGRAPH MODEL BASED FOR QUALITATIVE FDI DESIGN}

In this section, we first recall the Analytical Redundancy Relationship (ARR)algorithm based on Bond Graph model then we present our algorithm for ARR generation from a directed hypergraph model.

\subsection{Directed Hypergraph model-based FDI}

Based on causal and structural proprieties, a methodology to generate QARRs from a hypergraph model is proposed in this section.

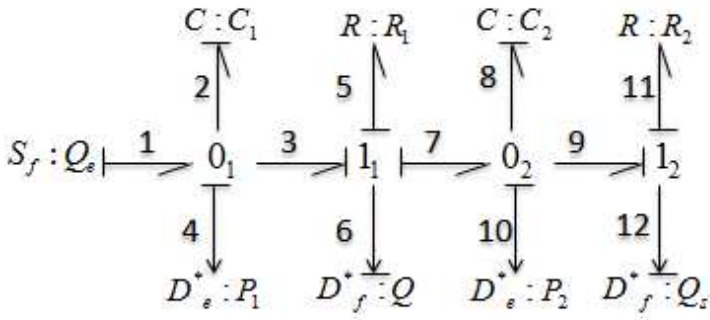

Fig. 7. BG model in preferred derivative causality

The generation of QRRs from the hypergraph modelk is based on the determination of causal hyperpaths that relate known variables. The known variables in the hyperpath are elements of the QRR.

The QRR generation proposed algorithm can be summarised as follows :

$\backslash * K$ the set of unknown variables $v_{i}$ in the directed hypergraph model* $^{*}$

Begin

$$
\begin{aligned}
& K=\{\text { unknown variables }\} \\
& j=1 \\
& \text { for } v_{i} \in K \text { do } \\
& Q R R_{j}=\emptyset
\end{aligned}
$$

A : Define $F S\left(v_{i}\right) \backslash *$ the set of hyperedges $e_{i}$ where $v_{i} \in T\left(e_{i}\right) *$

for $e_{j} \subseteq F S\left(v_{i}\right)$

if $\exists v_{k} \in H\left(e_{j}\right)$ a known variable then

$Q R R_{j}=\left\{Q R R, v_{k}\right\}$ go to $\mathbf{B}$

else $v_{i}=v_{k}$ return to $\mathbf{A}$

end if

end for

B : Define $B S\left(v_{i}\right) \backslash *$ the set of hyperedges $e_{i}$ where $v_{i} \in H\left(e_{i}\right)^{*}$

for $e_{j} \subseteq B S\left(v_{i}\right)$

if $\exists \quad v_{k} \in T\left(e_{j}\right)$ a known variable then

$Q R R_{j}=\left\{-v_{k}, Q R R_{j}\right\}$

else $v_{i}=v_{k}$ return to $\mathbf{B}$

end if

end for

$j=j+1$

end for

end

\subsection{Illustrative Example}

For illustration, consider again the example of the hydraulic two tanks systems shown on Figure 3, the Bond Graph model of the system is shown in (Figure 4).

To avoid initial condition problems which are not known in real processes, ARRs are directly generated from the BG model in derivative causality. Thus, the model in derivative causality given in Figure 7 is used for ARR generation.

Equations described $C_{1}, C_{2}, R_{1}$ and $R_{1}$, can be defined as follow :

$$
f_{2}=C_{1} \frac{d e_{2}}{d t}
$$




$$
\begin{gathered}
f_{8}=C_{2} \frac{d e_{8}}{d t} \\
e_{5}=\phi_{R_{1}}\left(f_{5}\right) \\
e_{11}=\phi_{R_{2}}\left(f_{11}\right)
\end{gathered}
$$

Where $\phi_{R_{2}}$ is a function of the valve characteristics. The capacities $C_{1}$ and $C_{2}$ are, respectively, equal to $\frac{\gamma C_{h_{1}}}{g}$ and $\frac{\gamma C_{h_{2}}}{g}$. g is the gravity. Constraints deduced from different junctions are :

$$
\begin{gathered}
J_{0_{1}}: f_{1}-f_{2}-f_{3}-f_{4}=0 \quad\left(f_{4}=0\right) \\
J_{1_{1}}: e_{4}-e_{5}-e_{6}-e_{7}=0 \quad\left(e_{6}=0\right) \\
J_{0_{2}}: f_{6}-f_{8}-f_{9}-f_{10}=0 \quad\left(f_{10}=0\right) \\
J_{1_{2}}: e_{10}-e_{11}-e_{12} \quad\left(e_{12}=0\right)
\end{gathered}
$$

To transform these constraints to ARR, all variables must be known. They will be expressed as function of known variables by covering causal paths. Hence, four fault indicators have to be generated as the following:

$$
\begin{gathered}
r_{1}: Q_{e}-C_{1} \frac{d P_{1}}{d t}-Q=0 \\
r_{2}: P_{1}-R_{1} Q-P_{2}=0 \\
r_{3}: Q-C_{2} \frac{d P_{2}}{d t}-Q_{s}=0 \\
r_{4}: P_{2}-R_{2} Q_{s}=0
\end{gathered}
$$

The associated quantitative fault signature matrix is given in Table (4.2). We can deduce that all faults are detected and isolated. The proposed algorithm is now used to generate the ARRs. The hypergraph model is shown in Figure 5 In this graph two variables are unknown $\dot{P}_{1}$ et $\dot{P}_{2} . E_{1}$ and $E_{2}$ (respectively $E_{3}$ and $E_{4}$ ) are adjacent to $\dot{P}_{1}$ (respectively to $\dot{P}_{2}$ ). $E_{1}$ is the incoming hyperedge and $E_{2}$ is the outgoing hyperedge to $\dot{P}_{1}$. We obtain the new hyperedge $E^{\prime}{ }_{1}=\left\{\left\{Q_{e}, Q\right\},\left\{P_{1}\right\}\right\}$. Likewise for $\dot{P}_{2}$ we obtain $E^{\prime}{ }_{2}=\left\{\left\{Q_{s}, Q\right\},\left\{P_{2}\right\}\right\}$. Vertices in $E_{1}^{\prime}$ represent the variables of the first Qualitative Redundancy Relationship $Q R R_{1}$ and vertices in $E_{2}^{\prime}$ are those of the second Qualitative Redundancy Relationship $Q R R_{2}$. The associated qualitative fault signature matrix is given in Table (4.2).

Table 1. Quantitative fault signature Matrix

\begin{tabular}{|c|c|c|c|c|c|c|}
\hline & QRR1 & QRR2 & QRR3 & QRR4 & $D$ & $I$ \\
\hline \hline$Q$ & 1 & 1 & 1 & 0 & 1 & 1 \\
\hline$Q_{s}$ & 0 & 0 & 1 & 1 & 1 & 1 \\
\hline$P_{1}$ & 1 & 1 & 0 & 0 & 1 & 1 \\
\hline$P_{2}$ & 0 & 1 & 1 & 1 & 1 & 1 \\
\hline
\end{tabular}

Table 2. Qualitative fault signature matrix

\begin{tabular}{|c|c|c|c|c|}
\hline & QRR1 & QRR2 & $D$ & $I$ \\
\hline \hline$Q$ & -1 & -1 & 1 & 1 \\
\hline$Q_{s}$ & 0 & -1 & 1 & 1 \\
\hline$P_{1}$ & 1 & 0 & 1 & 1 \\
\hline$P_{2}$ & 0 & 1 & 1 & 1 \\
\hline
\end{tabular}

By comparing these two tables, we note that the number of redundancy relationship are reduced and all faults are still isolable. Moreover, if one use the BG-hypergraph transformations there is no need to transform the Bond Graph model to the derivative causality. The BG-based method provides an intuitive and direct way to derive ARRs from the BG model. However, the total number of derived ARRs may not be optimum. This case study shows that although BG-based method provides good results in the diagnosis of chemical processes, the total number of arrs derived by this method may not be optimum.

\section{CASE STUDY}

\subsection{Hypergraph representation}

The case study deals with a reaction which has a second-order reaction rate between acid acetic $\mathrm{C}_{2} \mathrm{H}_{4} \mathrm{O}_{2}$ and ethanol $\mathrm{C}_{2} \mathrm{H}_{6} \mathrm{O}$ to produce ethyl acetate $\mathrm{C}_{4} \mathrm{H}_{8} \mathrm{O}_{2}$ and water $\mathrm{H}_{2} \mathrm{O}$. This reaction is represented by:

$$
\mathrm{C}_{2} \mathrm{H}_{4} \mathrm{O}_{2}+\mathrm{C}_{2} \mathrm{H}_{6} \mathrm{O} \underset{\mathrm{A}_{r}}{\stackrel{A_{f}}{\rightleftharpoons}} \mathrm{C}_{4} \mathrm{H}_{8} \mathrm{O}_{2}+\mathrm{H}_{2} \mathrm{O}
$$

It is a widely used reaction in engineering laboratories because it illustrates the theory of reactor very easily. In the sequel $A, B, C$ and $D$ stand for acid acetic, ethanol,ethyl acetate and water [6]. This reaction can be written in the general form as:

$$
v_{A} A+v_{B} B \underset{A_{r}}{\stackrel{A_{f}}{\rightleftharpoons}} v_{C} C+v_{D} D
$$

where $v_{i}$ (for $i=A, B, C, D$ ) are the stoichiometric coefficients. In our case these coefficients are equal to one because the esterification is one-to-one reaction.

Figure 8 presents the directly generation of the corresponding hypergraph $H=(V, E)$ with :

$V=\left\{\dot{n}_{1 A}, \dot{m}_{A}, \dot{n}_{A i}, \dot{n}_{A o}, \dot{n}_{A}, \dot{n}_{1 B}, \dot{m}_{B}, \dot{n}_{B i}, \dot{n}_{B o}, \dot{n}_{B}, \dot{n}_{1 C}, \dot{m}_{C}, \dot{n}_{C o}, \dot{n}_{C}\right.$, $\left.\dot{n}_{1 D}, \dot{m}_{D}, \dot{n}_{D o}, \dot{n}_{D}, \mu_{A}, \mu_{B}, \mu_{C}, \mu_{D}, A_{f}, A_{r}, A, J, T, \dot{S}\right\}$ and $E=\left\{E_{1}, E_{2}, \ldots, E_{23}\right\}$

where:

$$
\begin{aligned}
& E_{1}=\left(\left\{\dot{n}_{1 A}, \dot{n}_{A i}, \dot{n}_{A o}\right\},\left\{\dot{n}_{A}\right\}\right), E_{2}=\left(\left\{\dot{n}_{1 B}, \dot{n}_{B i}, \dot{n}_{B o}\right\},\left\{\dot{n}_{B}\right\}\right) \\
& E_{3}=\left(\left\{\dot{n}_{1 C}, \dot{n}_{C o}\right\},\left\{\dot{n}_{C}\right\}\right), E_{4}=\left(\left\{\dot{n}_{1 D}, \dot{n}_{D o}\right\},\left\{\dot{n}_{D}\right\}\right) \\
& E_{5}=\left(\{J\},\left\{\dot{n}_{1 A}\right\}\right), E_{6}=\left(\{J\},\left\{\dot{n}_{1 B}\right\}\right), E_{7}=\left(\{J\},\left\{\dot{n}_{1 C}\right\}\right), \\
& E_{8}=\left(\{J\},\left\{\dot{n}_{1 D}\right\}\right), E_{9}=\left(\left\{\dot{m}_{A}\right\},\left\{\dot{n}_{A i}\right\}\right), E_{10}=\left(\left\{\dot{m}_{B}\right\},\left\{\dot{n}_{B i}\right\}\right) \\
& E_{11}=\left(\left\{\dot{m}_{A}, \dot{n}_{A}\right\},\left\{\dot{n}_{A o}\right\}\right), E_{12}=\left(\left\{\dot{m}_{B}, \dot{n}_{B}\right\},\left\{\dot{n}_{B o}\right\}\right), \\
& E_{13}=\left(\left\{\dot{m}_{C}, \dot{n}_{C}\right\},\left\{\dot{n}_{C o}\right\}\right), E_{14}=\left(\left\{\dot{m}_{D}, \dot{n}_{D}\right\},\left\{\dot{n}_{D o}\right\}\right), \\
& E_{15}=\left(\left\{\dot{n}_{A}\right\},\left\{\mu_{A}\right\}\right), E_{16}=\left(\left\{\dot{n}_{B}\right\},\left\{\mu_{B}\right\}\right), E_{17}=\left(\left\{\dot{n}_{C}\right\},\left\{\mu_{C}\right\}\right) \\
& E_{18}=\left(\left\{\dot{n}_{D}\right\},\left\{\mu_{D}\right\}\right), E_{19}=\left(\left\{\mu_{A}, \mu_{B}\right\},\left\{A_{f}\right\}\right), \\
& E_{20}=\left(\left\{\mu_{C}, \mu_{D}\right\},\left\{A_{r}\right\}\right), E_{21}=\left(\left\{A_{f}, A_{r}\right\},\{A\}\right), \\
& E_{22}=(\{A, J\},\{\dot{S}\}), E_{23}=\left(\left\{\dot{n}_{A}, \dot{n}_{B}, \dot{n}_{C}, \dot{n}_{D}\right\},\{J\}\right)
\end{aligned}
$$




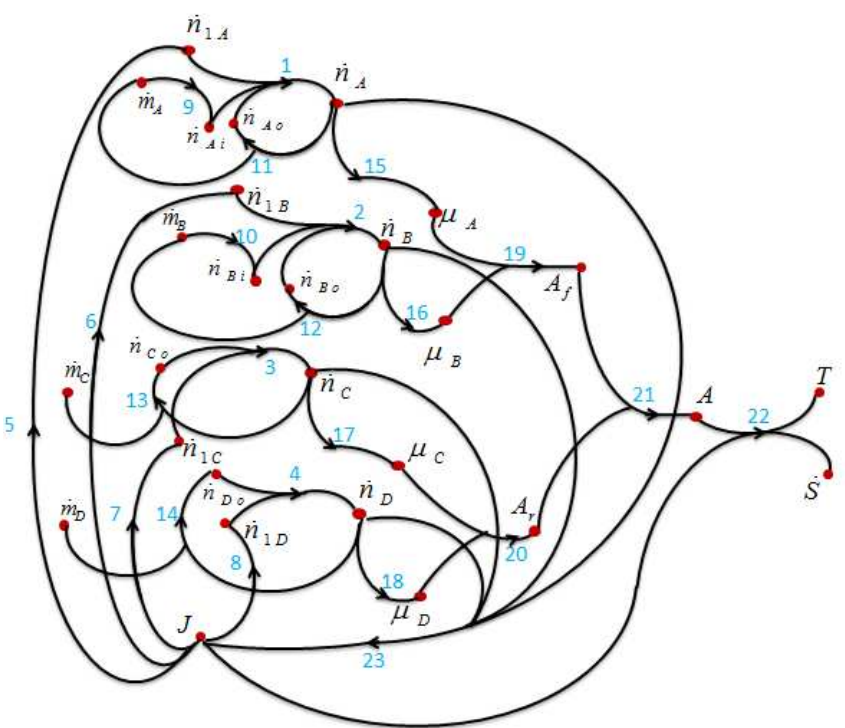

Fig. 8. Global hypergraph model $H$

The corresponding incidence matrix $B$ of the hypergraph $H$ is defined as follows :

$$
b_{i j}=\left\{\begin{array}{l}
-1 \quad \text { if } \quad v_{i} \in T\left(E_{j}\right) \\
1 \quad \text { if } \quad v_{i} \in H\left(E_{j}\right) \\
0 \text { otherwise }
\end{array}\right.
$$

The BGs have been successfully used for modeling different kinds of systems involving multiphysical phenomena [4]. Hence, an easier understand of the overall system. Figure (9) presents the proposed BG model of the considered esterification [6].Based on BGhypergraph analogy, the automatically generation of the hypergraph model $H_{B G}$ of the chemical kinetics is deduced directly from a Bond graph model as shown in Figure (10].

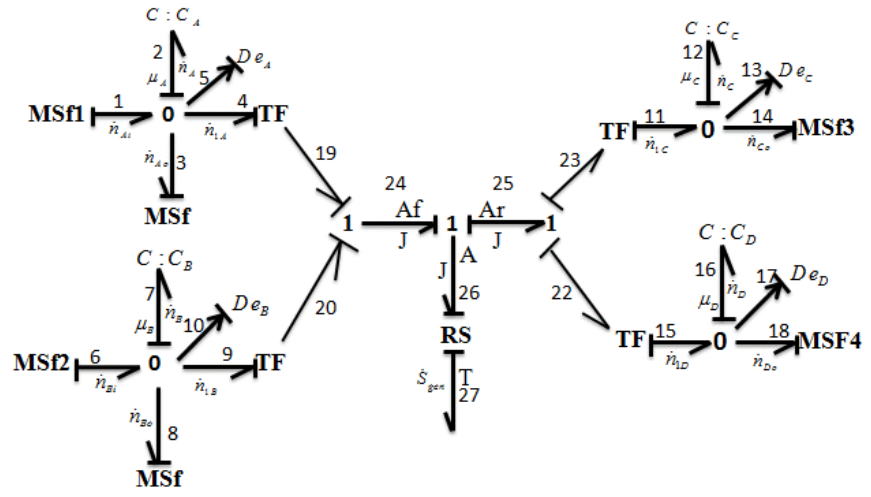

Fig. 9. Bond Graph model

One may easily check even the hypergraph $H_{B G}$ presents the same incidence matrix. Thus, both of the hypergraphs model refer to the same chemical reaction kinetics.

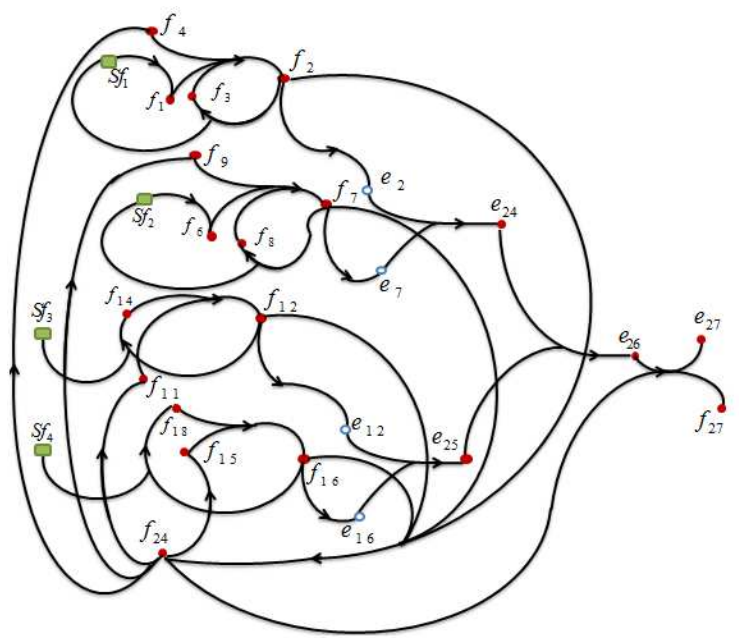

Fig. 10. deduced hypergraph model

\subsection{Fault monitoring and main results}

The chemical reaction Bond graph model in derivative causality is given (Figure (11)). From 0 junctions 4 ARRs can be obtained. The

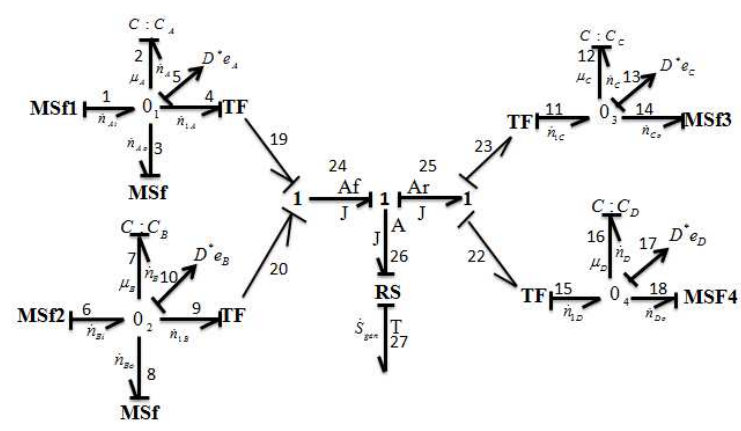

Fig. 11. Chemical reaction BG model in deriatie causality

candidate ARR generated from junction $0_{1}$ is :

$$
A R R_{1}=f_{1}-f_{2}-f_{3}-f_{4}=0
$$

where $f_{1}=M S f=\dot{n}_{A i}=\frac{\dot{m}_{A}}{M_{A}}, f_{2}=C_{A} \frac{d e_{2}}{d t}, f_{3}=M S f=\dot{n}_{A o}=n_{A} \frac{\dot{m}_{A}}{\rho V}$ and $f_{4}=v_{A} J$.

The unknown variable $f_{2}$ is eliminated by covering the causal path: $f_{2} \rightarrow \phi_{C_{A}} \rightarrow e_{2} \rightarrow \tilde{D} e_{A}: \mu_{A}$

The first ARR is then deduced substituting unknown variable in Eq. 46:

$$
\begin{aligned}
A R R_{1} & =\dot{n}_{A i}-\frac{V}{R T_{r}} \exp \left(\frac{\mu_{A}-\mu_{A 0}}{R T}\right)-n_{A} \frac{\dot{m}_{A}}{\rho V}-v_{A} J \\
& =\frac{\dot{m}_{A i}}{M_{A}}-\frac{V}{R T_{r}} \exp \left(\frac{\mu_{A}-\mu_{A 0}}{R T_{r}}\right)-n_{A} \frac{\dot{m}_{A}}{\rho V}-v_{A} r_{f}\left(1-\exp \left(\frac{A}{R T_{r}}\right)\right) V \\
& =0
\end{aligned}
$$


$\mu_{A 0}$ is the standard chemical potential.

From junction $\mathrm{O}_{2}$, we obtain :

$$
\begin{aligned}
A R R_{2} & =\dot{n}_{B i}-\frac{V}{R T_{r}} \exp \left(\frac{\mu_{B}-\mu_{B 0}}{R T}\right)-n_{B} \frac{\dot{m}_{B}}{\rho V}-v_{B} J \\
& =\frac{\dot{m}_{B i}}{M_{B}}-\frac{V}{R T_{r}} \exp \left(\frac{\mu_{B}-\mu_{B 0}}{R T_{r}}\right)-n_{B} \frac{\dot{m}_{B}}{\rho V}-v_{B} r_{f}\left(1-\exp \left(\frac{A}{R T_{r}}\right)\right) V \\
& =0
\end{aligned}
$$

From junction $0_{3}$, we have

$$
f_{11}-f_{12}-f_{14}=0
$$

where $f_{11}=v_{C} J=v_{C} r_{f}\left(1-\exp \left(\frac{A}{R T_{r}}\right)\right) V, f_{12}=C_{C} \frac{d e_{12}}{d t}$ and $f_{14}=M S f 3=\dot{n}_{C o}=n_{C} \frac{\dot{m}_{C}}{\rho V}$.

he unknown variable $f_{12}$ is deduced from the causal path: $f_{12} \rightarrow \phi_{C_{C}} \rightarrow e_{12} \rightarrow \tilde{D} e_{C}: \mu_{C}$

Thus the third ARR is :

$$
\begin{aligned}
A R R_{3} & =v_{C} r_{f}\left(1-\exp \left(\frac{A}{R T_{r}}\right)\right) V-n_{C} \frac{\dot{m}_{C}}{\rho V}-\frac{V}{R T_{r}} \exp \left(\frac{\mu_{C}-\mu_{C 0}}{R T_{r}}\right) \\
& =0
\end{aligned}
$$

And from junction $0_{4}$, we obtain :

$$
\begin{aligned}
& A R R_{4}=v_{D} r_{f}\left(1-\exp \left(\frac{A}{R T_{r}}\right)\right) V-n_{D} \frac{\dot{m}_{D}}{\rho V}-\frac{V}{R T_{r}} \exp \left(\frac{\mu_{D}-\mu_{D 0}}{R T_{r}}\right) \\
& \quad=0
\end{aligned}
$$

Table 5.2] gives the fault signature matrix of the studied chemical reaction :

Table 3. FSM deduced from the BG model

\begin{tabular}{|c|c|c|c|c|c|c|}
\hline & ARR1 & ARR2 & ARR3 & ARR4 & $D$ & $I$ \\
\hline \hline$\dot{m}_{A}$ & -1 & 0 & 0 & 0 & 1 & 1 \\
\hline$\dot{m}_{B}$ & 0 & -1 & 0 & 0 & 1 & 1 \\
\hline$\dot{m}_{C}$ & 0 & 0 & -1 & 0 & 1 & 1 \\
\hline$\dot{m}_{D}$ & 0 & 0 & 0 & -1 & 1 & 1 \\
\hline$\mu_{A}$ & 1 & 0 & 0 & 0 & 1 & 1 \\
\hline$\mu_{B}$ & 0 & 1 & 0 & 0 & 1 & 1 \\
\hline$\mu_{C}$ & 0 & 0 & 1 & 0 & 1 & 1 \\
\hline$\mu_{D}$ & 0 & 0 & 0 & 1 & 1 & 1 \\
\hline
\end{tabular}

The hypergraph model of the studied chemical reaction is given in Figure 8 The set of unknown variables is $K=\left\{\dot{n}_{A}, \dot{n}_{B}, \dot{n}_{C}, \dot{n}_{D}\right\}$. step 1:FS $\left(\dot{n}_{A}\right)=\left\{e_{15}, e_{23}\right\}$, for $e_{15}$, the chemical potential $\mu_{A}$ is the known variable in $H\left(e_{15}\right)$ thus $P=\left\{\mu_{A}\right\}$

$B S\left(\dot{n}_{A}\right)=\left\{e_{1}\right\}$, the known variable in $T\left(e_{1}\right)$ is the molar flow $\dot{m}_{A}$ and $P_{1}=\left\{\mu_{A},-\dot{m}_{A}\right\}$. We proceed in the same manner for the others variables in $\mathrm{K}$. We obtain $P_{2}=\left\{\mu_{B},-\dot{m}_{B}\right\}, P_{3}=\left\{\mu_{C},-\dot{m}_{C}\right\}, P_{4}=$ $\left\{\mu_{D},-\dot{m}_{D}\right\}$. The obtained result is summarized in the following fault signature matrix (Table $(5.2)$ )

\section{CONCLUSION AND OUTLOOK}

The hypergraph provides an important approach for modelling chemical reaction kinetics. This model was developed based on the basic energy and material balances. A difficulty is that even if a hypergraph model can be built from mathematical equations ([1]), precise equations are usually unavailable. The hypergraph model is then built on the bases of the process knowledge using the Bond Graph model developed from deep physical understanding of the complex system, thus avoiding the complexity of numerical calculations.
Table 4. FSM deduced from the hypergraph model

\begin{tabular}{|c|c|c|c|c|c|c|}
\hline & QRR1 & QRR2 & QRR3 & QRR4 & $D$ & $I$ \\
\hline \hline$\dot{m}_{A}$ & -1 & 0 & 0 & 0 & 1 & 1 \\
\hline$\dot{m}_{B}$ & 0 & -1 & 0 & 0 & 1 & 1 \\
\hline$\dot{m}_{C}$ & 0 & 0 & -1 & 0 & 1 & 1 \\
\hline$\dot{m}_{D}$ & 0 & 0 & 0 & -1 & 1 & 1 \\
\hline$\mu_{A}$ & 1 & 0 & 0 & 0 & 1 & 1 \\
\hline$\mu_{B}$ & 0 & 1 & 0 & 0 & 1 & 1 \\
\hline$\mu_{C}$ & 0 & 0 & 1 & 0 & 1 & 1 \\
\hline$\mu_{D}$ & 0 & 0 & 0 & 1 & 1 & 1 \\
\hline
\end{tabular}

The cause-effect structure impled by the hypergraph makes it amenable for diagnosis, where the goal is to determine the causes of observed abnormal situation. The application of the proposed approach has been fulfilled through the example of a reaction which has a second-order reaction rate.

\section{REFERENCES}

[1] Henda Ajemni, Rafika El Harrabi, and Mohamed Naceur Abdelkrim. Modeling of chemical reaction kinetics using hypergraph tools. In Electrical Sciences and Technologies in Maghreb (CISTEM), 2014 International Conference on, pages 1-6. IEEE, 2014.

[2] Giorgio Ausiello, Roberto Giaccio, Giuseppe F Italiano, and Umberto Nanni. Optimal traversal of directed hypergraphs. 1992.

[3] Claude Berge and Edward Minieka. Graphs and hypergraphs, volume 7. North-Holland publishing company Amsterdam, 1973.

[4] G Dauphin-Tanguy. Les bond graphs, hermes science europe ltd., paris, france, 2000. Technical report, ISBN 2-7462-01585.

[5] M Ederer and ED Gilles. Thermodynamic constraints in kinetic modeling: Thermodynamic- kinetic modeling in comparison to other approaches. Engineering in Life Sciences, 8(5):467-476, 2008.

[6] Rafika El Harabi. Supervision des processus chimiques à base de modèles Bond Graphs. PhD thesis, Lille 1, 2011.

[7] Carlos Heny, Daniel Simanca, and Marisol Delgado. Pseudobond graph model and simulation of a continuous stirred tank reactor. Journal of the Franklin Institute, 337(1):21-42, 2000.

[8] Steffen Klamt, Utz-Uwe Haus, and Fabian Theis. Hypergraphs and cellular networks. PLoS computational biology, 5(5):e1000385, 2009.

[9] Dan Klein and Christopher D Manning. Parsing and hypergraphs. In New developments in parsing technology, pages 351-372. Springer, 2005.

[10] B Ould-Bouamama, Rafika El Harabi, Mohamed Naceur Abdelkrim, and MK Ben Gayed. Bond graphs for the diagnosis of chemical processes. Computers \& chemical engineering, 36:301-324, 2012.

[11] Oleg N Temkin, Andrew V Zeigarnik, and DG Bonchev. Chemical reaction networks: a graph-theoretical approach. CRC Press, 1996.

[12] Andrew V Zeigarnik. On hypercycles and hypercircuits in hypergraphs. Discrete Mathematical Chemistry, 51:377-383, 2000. 